\title{
The effect of repetition upon the serial learning of familiar items
}

\begin{abstract}
JOHN F. CATALANO, State University of New York,
\end{abstract} Cortland, N.Y. 13045

Two experiments were conducted in which serial learning of familiar names with standard repetition was compared to learning under a modified procedure where repetition was eliminated. In the first experiment, the modification involved removal of incorrectly anticipated items and the substitution of new items. In the second experiment the positions of incorrect items were interchanged. In both experiments learning with repetition was significantly faster.

Rock (1957) presented evidence that associations are formed in an "all-or-none" manner and proposed that repetition is unnecessary for the acquisition of associations. Rock's unique and original modification of standard paired-associate procedure involved the removal of unlearned pairs and the substitution of new pairs. It was found that learning under substitution occurred at the same rate as under the standard procedure. However, in serial learning of nonsense syllables, Brosgole, Hansen \& Contino (1968) found that learning is significantly more difficult when incorrectly anticipated items are replaced with new items, as compared to the standard procedure of repeatedly presenting the same list.

The present paper is concerned with determining the role of repetition in serial learning, but here $S$ is required to learn familiar items rather than nonsense syllables. An unfortunate consequence of the substitution procedure is that $S$ s are required to become familiar with new items and to form associations on a single trial. Under the standard condition, even though an item is not correctly anticipated, it does become increasingly familiar with repetition. Such a differential familiarity may serve to mask the fact that, once familiar, associations are indeed formed in an all-or-none manner as Rock contends.

\section{EXPERIMENT 1}

The purpose of this experiment is to determine whether the rate of serial learning of familiar names is affected by the substitution of new items for incorrectly anticipated items.

Procedure

The learning materials consisted of a pool of 200 male names. Variations of a name were avoided in order to provide discrete and independent items. Each name was typed on a $3 \times 5$ in. index card and presented manually by $E$ who was seated behind a screen. Two learning conditions were employed, substitution and repetition. On the first trial of the substitution condition, 10 items were presented to $S$ at $5-\mathrm{sec}$ intervals. $\mathrm{S}$ was instructed to read aloud each item presented. On the second trial, $S$ was asked to anticipate the items in the order presented. $\mathrm{S}$ was allowed $5 \mathrm{sec}$ to respond. If $\mathrm{S}$ anticipated correctly he was shown the item and asked to anticipate the next item. If $S$ did not respond or was incorrect, the item which was to appear was removed from the list and replaced with a new item, which was shown to S. Only those items which were anticipated correctly remained from trial to trial. This procedure continued until $\mathrm{S}$ correctly anticipated the entire list.

The repetition condition followed standard serial anticipation procedure, the criterion of learning being one correct anticipation of the entire list. Ten male names were used which differed from any used under the substitution condition. As in the substitution condition, $5 \mathrm{sec}$ were allowed for anticipation. Each $\mathrm{S}$ was tested under both conditions.
Order of presentation was balanced over all Ss. Each $\mathrm{S}$ was given a few preliminary trials prior to each condition, with females' names. There was a 10 -min rest period between conditions.

\section{Subjects}

Five male and four female undergraduate students served as Ss. Their ages ranged from 18-24; their mean age was 19.4.

\section{Results and Discussion}

The mean number of trials to reach criterion under the substitution and repetition conditions were 6.1 and 4.0, respectively. The Wilcoxon Matched Pairs Signed Ranks test revealed that learning with repetition was significantly superior to the substitution method ( $\mathrm{T}=1.5, \mathrm{~N}=8, \mathrm{p}<.02)$.

Observation of Ss' behavior and their comments indicated that the substitution condition provided the more difficult learning task. Ss were sometimes disturbed by the appearance of a new item when they were confident they had been correct. Under the substitution condition $S$ was exposed to many more items than under the repetition condition. It was possible that an $S$ requiring only five trials to reach criterion under substitution was presented with over three times the number of items presented under the repetition condition. Figure 1 shows a comparison of the errors which occurred under the two conditions. The repetition curve is typically bow-shaped, showing the serial-position effect. The substitution condition markedly increases in error frequency beyond the fourth position. Learning seems to have been accomplished through a chaining process, i.e., attaching a new item to those previously learned.

The results of this experiment show that serial learning of familiar items is superior with repetition. This finding is not in accord with an all-or-none theory of learning.

\section{EXPERIMENT 2}

The superiority of learning with repetition which occurred in Experiment 1 may have simply been due to the fact that under this procedure $\mathrm{S}$ was presented with only 10 items, whereas under substitution was exposed to many more.

The difference between the number of items $S$ experienced, rather than repetition per se, may have been responsible for the different rates of learning.

The purpose of Experiment 2 was, again, to determine the effect of repetition upon serial learning, but here the number of items to be learned was held equal for the repetition and a nonrepetition condition. This was accomplished by changing the positions of unlearned items within a serial list. Ebenholtz

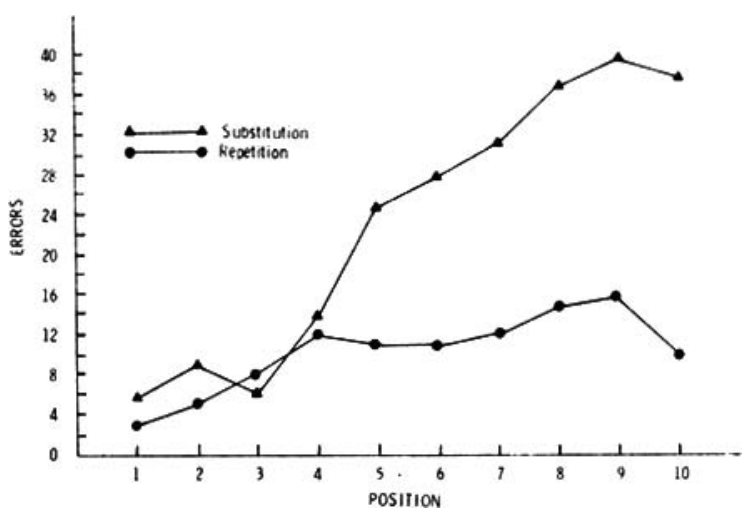

Fig. 1. Error frequency at each position in Experiment 1. 


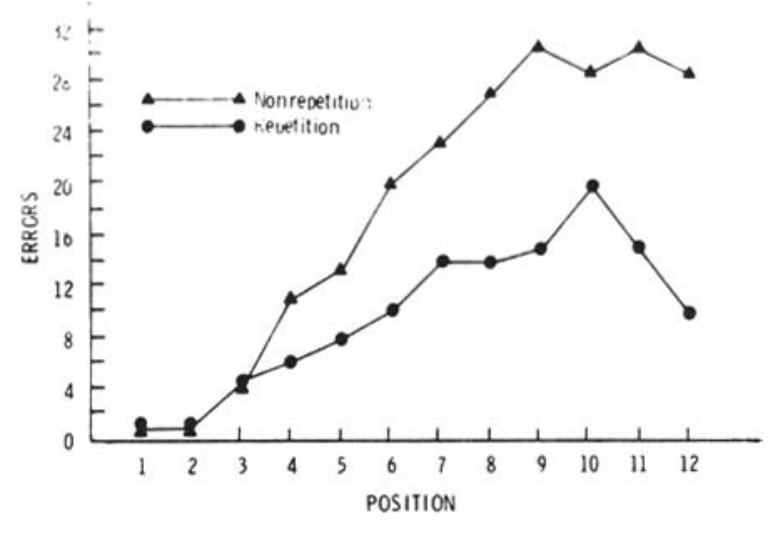

Fig. 2. Error frequency at each position in Experiment 2.

(1963) has shown that serial learning can occur quite readily when sequential learning is precluded. Ebenholtz maintains that serial learning is to a great extent based upon the piecing together of items or parts of a list that have been associated with specific positions in the series. The stimulus for the formation of an association is position, and not the preceding item as is commonly assumed.

If learning is indeed all-or-none rather than incremental, and if position associations are as important as Ebenholtz suggests, then one would expect that changing the position of unlearned items should have no effect upon acquisition, since no "trace" of the association has been formed. If, on the other hand, serial learning occurs incrementally, associations, even though subthreshold, are formed with every presentation. Failure to respond correctly does not mean that no learning has occurred according to the incremental view. Therefore, changing the location of an incorrect item will work against any subthreshold associations that have been formed and learning will be relatively difficult.

\section{Procedure}

A serial recall procedure was employed. In the nonrepetition condition, 12 items were presented to each $S$ for $5 \mathrm{sec}$. After seeing the list, $S$ was asked to recall the list in the order that it was just presented. $S$ was told that items recalled correctly would remain in the same position, while incorrect items would be shuffled and their positions interchanged from trial to trial. Responses were scored as correct if $S$ could repeat the items in sequence or could identify an item with a specific position. If an error was made with a previously correct item its position was changed. Ss were allowed $20 \mathrm{sec}$ to recall the entire list. The criterion of learning was one correct trial. The repetition condition was similar to the nonrepetition condition, except that all items occupied the same position from trial to trial. Each $S$ served under both conditions, and order was balanced. The learning material consisted of 12 male and 12 female names typed on $3 \times 5$ in. index cards. For each $S$, male names were used for one condition and female names for the other, balanced over all Ss. There was a 10-min rest period between conditions.

\section{Subjects}

Ten undergraduate students, five males and five females served as Ss. Their ages ranged from 18 to 23 with a mean of 19.6.

\section{Results and Discussion}

The mean number of trials to criterion for the nonrepetition and repetition conditions were 6.0 and 3.5. The Wilcoxon test showed these differences to be significant $(T=0, N=8$, $\mathrm{p}<.01$ ). In Fig. 2 error frequency at each position is plotted. The curves coincide for the first three positions. From the fourth position on, fewer errors occurred with repetition.

Under the conditions of this experiment, repetition facilitated learning. However, it should be noted that this experiment is a fair test of the all-or-none hypothesis only to the extent that serial learning is based upon the formation of position associations. If, on the other hand, sequential associations are of primary importance for serial learning to occur, then an item, whether recalled or not, must occupy a permanent position in order to serve as a stimulus for the next item. If such is the case, then changing positions would have resulted in the disruption of any associations that might have been formed or a single trial.

The results of these experiments, in addition to those of Brosgole et al, lead to the conclusion that repetition facilitates serial learning. While it may be true that serial learning can occur without repetition, it may do so at great cost to the learner in terms of effort and frustration. However, not all learners find repetition to be advantageous. Two Ss in Experiment 1 and two Ss in Experiment 2 learned at the same rate under both conditions and might be regarded as "all-or-none learners." Postexperimental interviews revealed that these Ss employed a strategy of concentrating only upon material within their memory span, ignoring the remaining material for that trial.

It may be that the issue concerning the formation of associations focuses upon the wrong process, and that it would be much more fruitful to get a better understanding of the kinds of strategies employed in learning and under what conditions they are employed. Perhaps future research should seek to explain why a strategy that makes repetition appear to be necessary for the formation of associations is employed by the majority of individuals.

\section{REFERENCES}

BROSGOLE, L., HANSEN, K. H., \& CONTINO, A. F. Repetition and serial learning. Psy chonomic Science, in press.

EBENHOLTZ, S. M. Serial leaming: position learning and sequential associations. Journal of Experimental Psychology, 1963, 66, 353-362. ROCK, I. The role of repetition in associative learning. American Journal of Psychology, 1957, 70, 186-193. 